\title{
Recent developments of wide-bandgap semiconductor based UV sensors
}

\author{
A. BenMoussa ${ }^{\text {a,* }}$, A. Soltani ${ }^{b}$, U. Schühle ${ }^{c}$, K. Haenen ${ }^{\text {d }}$, Y.M. Chong ${ }^{\text {e }}$, W.J. Zhang ${ }^{\text {e }}$, R. Dahal ${ }^{\text {f }}$, J.Y. Lin ${ }^{\text {f,g }}$,

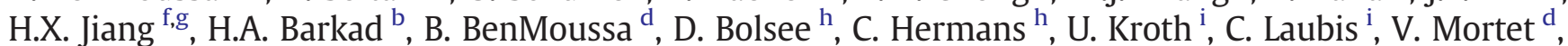 \\ J.C. De Jaeger ${ }^{\text {b }}$, B. Giordanengo ${ }^{\text {a }}$, M. Richter ${ }^{\text {i }}$, F. Scholze ${ }^{\text {i }}$, J.F. Hochedez ${ }^{\text {a }}$ \\ a Royal Observatory of Belgium, Solar Terrestrial Center of Excellence (STCE), Circular Avenue 3, B-1180 Brussels, Belgium \\ b Institut d'Electronique, de Microélectronique et de Nanotechnologie (IEMN/CNRS 8520), Avenue Poincaré, cité scientifique F-59652 Villeneuve d'Ascq, France \\ ${ }^{\mathrm{c}}$ Max-Planck-Institut für Sonnensystemforschung, D-37191 Katlenburg-Lindau, Germany \\ d Hasselt University, Institute for Materials Research (IMO), Wetenschapspark 1, B-3590 Diepenbeek, Belgium \\ e Center of Super-Diamond and Advanced Films (COSDAF) and Department of Physics and Materials Science, City University of Hong Kong, Hong Kong SAR, China \\ ${ }^{\mathrm{f}}$ Department of Physics, Kansas State University, Manhattan, Kansas 66506-2601, USA \\ g Nano-Tech Center and Dept. of ECE, Texas Tech University, Lubbock, TX 79409-3102, USA \\ ${ }^{\mathrm{h}}$ Belgium Institute for Space Aeronomy (BISA), Circular Avenue 3, B-1180 Brussels, Belgium \\ ${ }^{i}$ Physikalisch-Technische Bundesanstalt (PTB), Abbestr. 2-12, D-10587 Berlin, Germany
}

\section{A R T I C L E I N F O}

Available online 27 November 2008

Keywords:

UV photodetector

MSM

WBGM

Solarblind

\begin{abstract}
A B S T R A C T
Future missions for space astronomy and solar research require innovative vacuum ultraviolet (VUV) photodetectors. Present UV and VUV detectors exhibit serious limitations in performance, technology complexity and lifetime stability. New developments of metal-semiconductor-metal (MSM) solar-blind photodetectors based on diamond, cubic boron nitride (c-BN), and wurtzite aluminium nitride (AlN) are reported. In the wavelength range of interest, the characteristics of the MSM photodetectors present extremely low dark current, high breakdown voltage, and good responsivity. Diamond, c-BN, and AlN MSM photodetectors are sensitive and stable under UV irradiation. They show a $200 \mathrm{~nm}$ to $400 \mathrm{~nm}$ rejection ratio of more than four orders of magnitude and demonstrate the advantages of wide band gap materials for VUV radiation detection in space.
\end{abstract}

(C) 2008 Elsevier B.V. All rights reserved.

\section{Introduction}

Among solar observations and UV astronomy, solar-blind photodetectors have a variety of potential applications in the fields of automotive, military defense as well as in environmental and biological research. For the next envisaged space missions ESA and NASA planned to study the Sun, e. g., the Solar Orbiter, solar-blind photodetectors capable of operating at high temperatures and in harsh environments are a crucial ingredient.

A project called "Blind to Optical Light Detectors" (BOLD) [1] has been set up involving international partners with the goal of developing photodetectors using wide band gap materials (WBGM). The development of innovative UV photodetectors has progressed considerably during the last years [2-7]. However, they are still under development and further improvements of their performance characteristics are still needed. These are heavily dependent on the quality of the materials, used as the sensitive substrate.

Recently, high-quality diamond, cubic boron nitride (c-BN) and aluminium nitride (AIN) films were successfully grown. In this work,

\footnotetext{
* Corresponding author. Tel.: +32 237302 76; fax: +32 23730224 .

E-mail address: ali.benmoussa@oma.be (A. BenMoussa).

URL: http://bold.oma.be (A. BenMoussa).
}

we report on new metal-semiconductor-metal (MSM) photodetectors based on these new emerging semiconductors. The electrical characteristics (e. g., I-V curves) and the absolute radiometric responsivities are reported.

\section{Detector technology innovations}

\subsection{Motivation for utilizing a planar structure - MSM photodetectors}

Various criteria require consideration when designing a UV photodetector for space applications. These factors include, among others, the optical wavelength of interest (here 10-122 nm), semiconductor material composition and thicknesses, detector geometrical structure (device architecture), and electrode metal composition. For UV detection applications, many different constructive designs have been developed from single-pixel photodiode-type detectors, to imaging arrays of micro-pixel structures [8-10].

For the purpose of VUV radiation detection, the penetration depth $1 / \alpha(\lambda)$ of diamond and AlN materials derived from [11-17] drops drastically to extremely small values of the order of few nanometres. As shown in Fig. 1, the photons are being absorbed in the first atomic layers, leading to carrier recombination in the upper surface layer and consequently to a reduction of the sensitivity. 


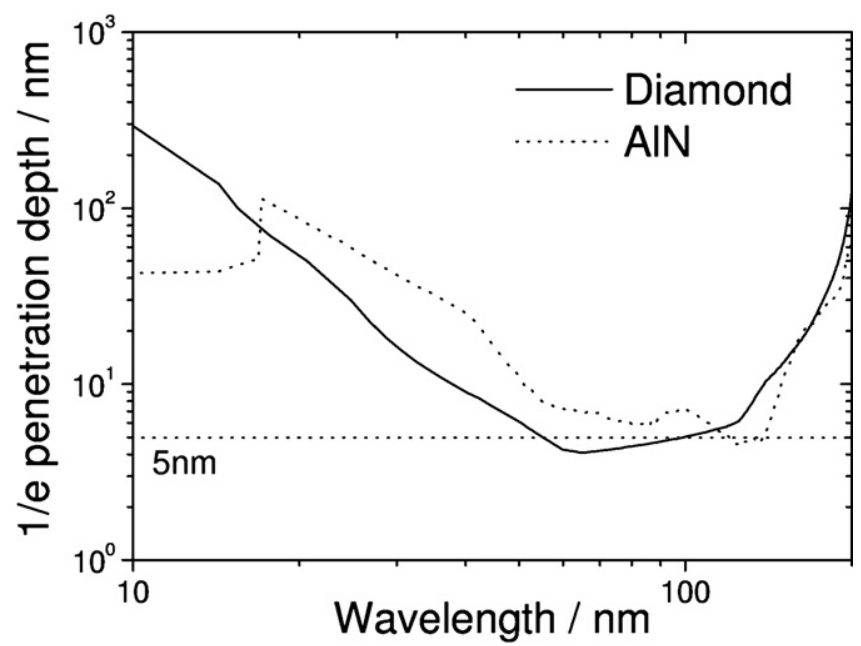

Fig. 1. Penetration depth versus wavelength in the range of $10 \mathrm{~nm}$ to $200 \mathrm{~nm}$ of diamond and AlN materials derived from different references [11-17].

Hence, it can be readily understood that it is crucial to optimize the photo-electron collection close to the surface with a planar configuration of the electrodes. Among different design structures, the MSM represents a simple, cost-effective photodetector design not only related to performance but also to ease the fabrication process. The MSM photodetector is built with coplanar structures, making their production simpler than $\mathrm{p}-\mathrm{i}-\mathrm{n}$ or Schottky diodes consisting of different layers with different doping constituents and concentrations. Their main limitations include relatively larger dark current since a bias is required that is nevertheless negligibly small for WBGM (see Fig. 5), and moderate external quantum efficiency (EQE) due to electrode shadowing, if they are not semi-transparent in the wavelength range of interest. Dedicated corrective design can however be foreseen [18].

Note that the reported diamond MSM photodetector has a different mode of operation than the c-BN and AlN MSM photodiodes since it is called a MSM photoconductor. The MSM photoconductor consists of a piece of semiconductor with two ohmic contacts where a potential difference is applied between them while the MSM photodiode is essentially two Schottky barrier diodes back-to-back fully depleted under sufficient bias. MSM photoconductors are different to MSM photodiodes in several important ways: current is carried both by minority and majority carriers in the photoconductor and the gain can be higher than unity depending primarily on the ratio of the minority carrier lifetime to the majority carrier transit time.

\subsection{Diamond MSM photoconductors}

Photodetectors based on diamond material have progressed only very recently due to difficulties in producing the high-quality thin films with sufficiently low defects and concentrations of n-type and p-type dopants [5].

In the frame, the PRoject for On-Board Autonomy 2 (PROBA2), the ESA micro-mission conceived for the purpose of demonstrating new technologies, has given the first opportunity to advance the use of innovative diamond detector technologies with the Large Yield RAdiometer (LYRA) [19], which is a solar VUV radiometer that will be launched in 2009 onboard PROBA-2. LYRA will be the first space assessment of the pioneering UV diamond detectors.

In this work, a new MSM device architecture to minimize shadowing by electrodes and increase the homogeneity of the diamond photodetectors is investigated. The reported device is designated MSM24-r. The circular metal fingers of the MSM24-r structure is designed to $2 \mu \mathrm{m}$ in width, with a spacing between the contacts of $5 \mu \mathrm{m}$. Metallization of ohmics contacts is Ti/Pt/Au with thicknesses of 20/10/200 nm, respectively. The electrodes supporting the metal fingers are $30 \mu \mathrm{m}$ in width. The Fig. 2 shows a photograph of the MSM24-r photodetector mounted inside its rectangular ceramic package. Homoepitaxial growth procedure and fabrication steps are detailed elsewhere $[20,21]$. The active area of the MSM24-r device has a diameter of $4.6 \mathrm{~mm}$ on top of a $5 \mathrm{~mm}$ diameter diamond substrate corresponding to an optical detection area of $16.6 \mathrm{~mm}^{2}$. Note that there is a general need in solar missions for large size $(\varnothing>3 \mathrm{~mm})$ photodetectors. This requirement brings the fabrication of photodetectors to a high level of complexity due to the homogeneity requirements on the large surfaces materials (roughness, defects). The large-size diamond photodetector demonstrates an added value relative to the state-of-the-art where typical areas of UV detectors are usually below $1 \mathrm{~mm}^{2}$.

\section{3. c-BN MSM photodiodes}

Cubic boron nitride (c-BN) films were deposited on diamondcoated silicon substrates in an electron-cyclotron-resonance microwave plasma CVD system using a $\mathrm{He}-\mathrm{Ar}-\mathrm{N}_{2}-\mathrm{BF}_{3}-\mathrm{H}_{2}$ gas mixture. The experimental details and the high crystallinity of the c-BN films deposited are reported elsewhere [22-25]. In this work, $1 \mu \mathrm{m}$ thick c-BN films were grown on the $4 \mu \mathrm{m}$ thick diamond interlayer (see Fig. 3). The previous studies indicate that c-BN films grew directly on diamond surfaces without a soft and humidity-sensitive non-cubic BN phase. As a result, the adhesion of c-BN films was improved significantly. Comprehensive characterization also illustrates that the c-BN films deposited by this method are in a polycrystalline columnar structure and have a high crystallinity and cubic phase
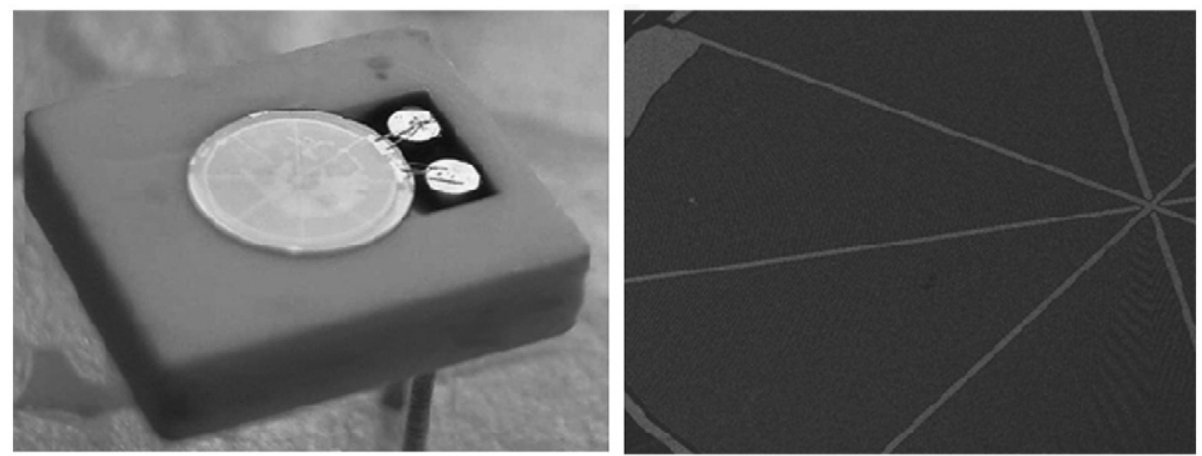

Fig. 2. Photographs of the new diamond MSM24-r photodetector mounted in a ceramic housing (close-up view on the right-hand side). 


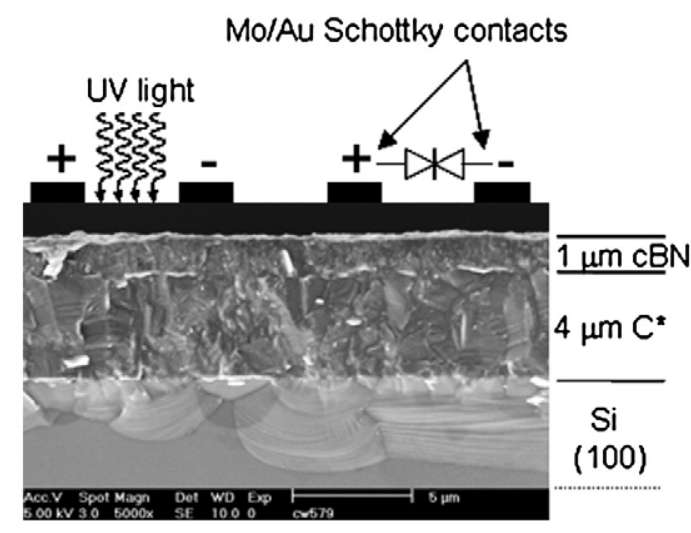

(a)

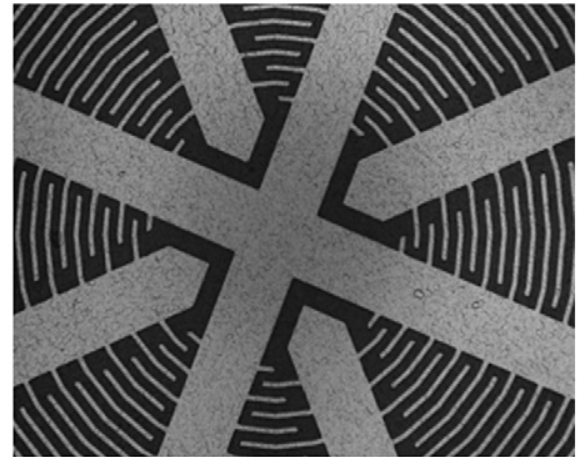

(b)

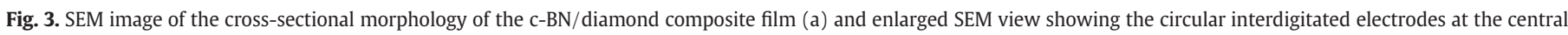
region (b).

purity $[23,24]$. The MSM photodiodes were fabricated by depositing patterned $\mathrm{Mo} / \mathrm{Au}(40 \mathrm{~nm} / 300 \mathrm{~nm}$ ) electrodes on the c-BN surface using electron beam deposition. After deposition, the samples are annealed at $400{ }^{\circ} \mathrm{C}$ for $15 \mathrm{~min}$ in an $\mathrm{N}_{2}$ atmosphere to improve the stability of Schottky electrode contacts. The diameter of the devices varies from $1 \mathrm{~mm}$ to $3 \mathrm{~mm}$. The finger width is $2 \mu \mathrm{m}$, and the inter-spacing between contact pads is $5 \mu \mathrm{m}$ (same circular design as the diamond MSM24-r photodetector) with an electrode fill factor (metal/c-BN area ratio) of less than 0.33 .

\subsection{AlN MSM photodiodes}

The aluminium nitride (AIN) MSM photodiodes are based on $1.5 \mu \mathrm{m}$ thick AlN epilayers grown on sapphire substrates using metal organic chemical vapor deposition (MOCVD). The Fig. 4(a) shows schematically the device layer structure. Heteroepitaxial growth procedure details are given elsewhere [26]. The quality of the AlN epilayers can be found from $[27,28]$ including very narrow line widths of X-ray diffraction (002) and (102) peaks with a dislocation densities of typically $5 \times 10^{9} \mathrm{~cm}^{-2}$. Three AlN MSM samples were connected together in parallel to form a larger device, resulting in an active area of $3 \times(0.1 \times 0.08) \mathrm{mm}^{2}$. The linear interdigitated fingers for Schottky contacts are designed to $2 \mu \mathrm{m}$ in width, with $4 \mu \mathrm{m}$ spacing between the contacts. Contacts are made by metallization of $\mathrm{Pt} / \mathrm{Au}$ with thicknesses of $10 \mathrm{~nm}$ and $200 \mathrm{~nm}$, respectively, followed by thermal annealing at a temperature of approximately $800{ }^{\circ} \mathrm{C}$. Recently, AlN Schottky diodes and AlN avalanche photodetectors have been demonstrated by the same research group [29,30].

\section{Experimental setup}

The optical measurements were carried out using a collimated and tuneable monochromatic light beam provided by a $100 \mathrm{~W}$ deuterium light source combined with a double monochromator. We could obtain the relative spectral response function between $175 \mathrm{~nm}$ and $400 \mathrm{~nm}$, but also check the rejection ratio and the stability of the signal at different bias voltages. The spectral resolution can be as low as $0.1 \mathrm{~nm}$ but typically it was $0.5 \mathrm{~nm}$ to $1 \mathrm{~nm}$. The beam from the deuterium lamp naturally diverges. It was collimated by the arrangement of condenser, pinhole and lenses which also improved the resulting beam homogeneity. Absolute calibration could be accomplished using calibrated silicon photodiodes (AXUV diodes from IRD Inc., USA) used as detectors for the spectrometers. Individual measurements were repeated to check stability and reproducibility. During the measurements, the beam stability was constantly monitored by alternating measurements of the sample and the reference diode.

In the VUV range ( $40 \mathrm{~nm}$ to $240 \mathrm{~nm}$ ), a synchrotron radiation campaign was carried out at the normal incidence $(\mathrm{NI})$ beamline of the Physikalisch-Technische Bundesanstalt (PTB) laboratory at the storage ring Berliner Elektronenspeichering-Gesellschaft für Synchrotronstrahlung (BESSY II). Details of the description of the PTB/BESSY II measuring stations can be found in [31,32]. The detector calibration facility provides a synchrotron beam, monochromatized by a NI monochromator with a spectral resolution of about $1 \%$ of the wavelength. To cover a wide spectral range from $40 \mathrm{~nm}$ to $240 \mathrm{~nm}$, different filters and beamline mirrors are used to suppress higher

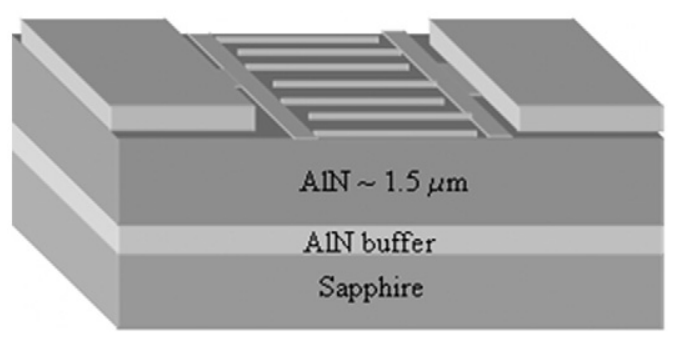

(a)

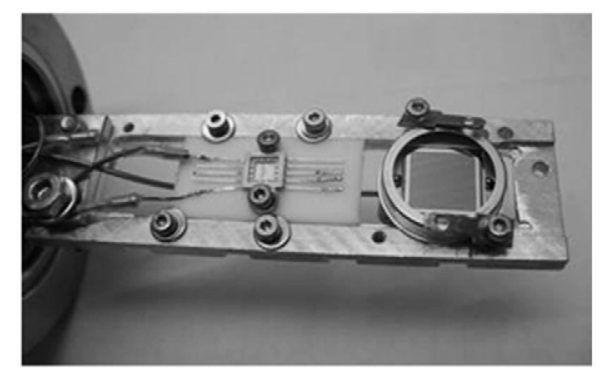

(b)

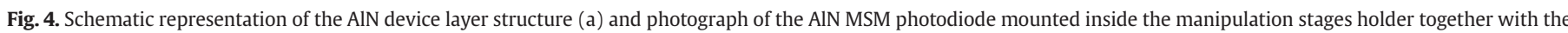
silicon reference photodiode at PTB-BESSY II synchrotron radiation beamline (b). 


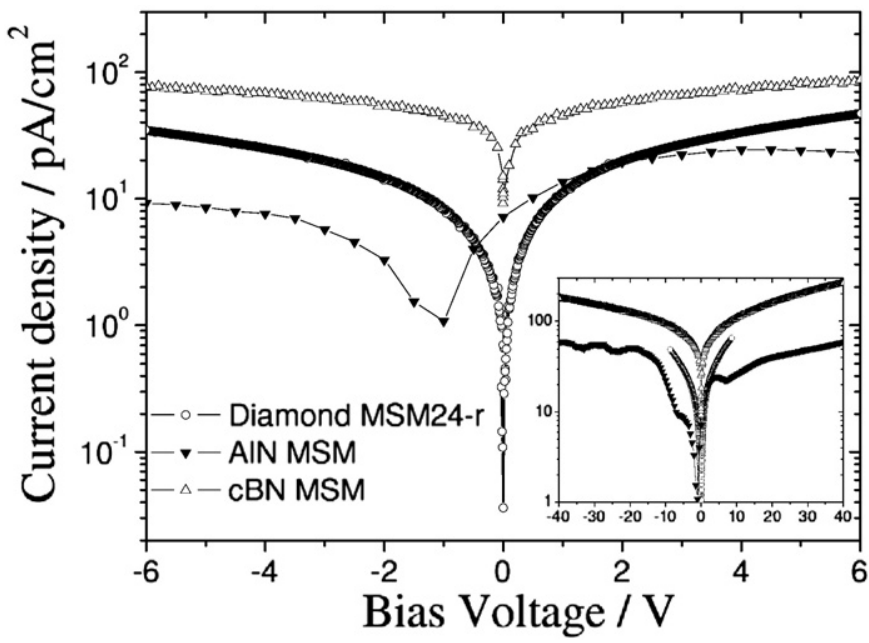

Fig. 5. Dark current density versus voltage characteristics of the diamond MSM24-r, c-BN, and AlN MSM photodetectors at room temperature between $-6 \mathrm{~V}$ and $+6 \mathrm{~V}$. The inset shows a large view of the dark current density between $-40 \mathrm{~V}$ and $+40 \mathrm{~V}$.

order contributions to the selected bandwidth. The detector calibration chamber at the end of the beamline is an ultrahigh vacuum chamber with manipulation stages permitting to raster the sample area and to toggle between test and reference detectors as shown in Fig. 4(b). The spot size of the beam at the location of the samples is approximately $1 \times 2 \mathrm{~mm}^{2}$, depending slightly on the chosen wavelength. The radiometric uncertainty of the measurements is estimated to be better than a few percent in absolute terms.

\section{Results and discussion}

\subsection{Electrical measurements}

The current density-voltage (I-V) curves were measured at room temperature and under $\mathrm{N}_{2}$ atmosphere. It must be noted that special care should be taken to keep the MSM detectors in a dry and inert environment to avoid water surface contamination and therefore an increase of the dark current. The goal of these measurements is not to compare the devices, since their electro-optical characteristics depend significantly on the surface properties of the active layer, on the applied voltage and on the geometrical design (width and distance between electrodes). Note also that the diamond MSM photodetector, as a photoconductor, has a different mode of operation. Thus we report only briefly these measurements here, which are depicted in Fig. 5.

The diamond MSM24-r photodetector shows a small dark current of $1.1 \mathrm{pA}$ at $+5 \mathrm{~V}$ bias ( $0.4 \mathrm{pA}$ before wire bonding) with ohmic Ti/Pt/ Au contacts. The I-V characteristic of the c-BN-based MSM photodiode reveals a Schottky contact between the Mo/Au electrodes and c-BN films. The device exhibits a low dark current of about $1.2 \mathrm{pA}$ to $-1.4 \mathrm{pA}$ at a bias voltage of $+/-30 \mathrm{~V}\left(160-190 \mathrm{pA} / \mathrm{cm}^{2}\right)$, respectively. For AlN MSM photodetectors, the I-V characteristic shows a negligibly small dark current of $13 \mathrm{fA}$ at $+/-30 \mathrm{~V}$ (i.e. $56 \mathrm{pA} / \mathrm{cm}^{2}$ ). The measured signal was stable over a wide temporal range.

\subsection{Absolute spectral responsivity}

The absolute spectral responsivity curves and more precisely the EQE of the MSM photodetectors are shown in Fig. 6. For clarity, the estimated error bars to the data points are not plotted. When synchrotron measurements are possible, the data measured on a relative scale (from $175 \mathrm{~nm}-400 \mathrm{~nm}$ ) are matched to the absolute data from PTB/BESSY II (40 nm-240 nm).
As seen in Fig. 6, the EQE of the MSM detectors show the band edges around $225 \mathrm{~nm}, 203 \mathrm{~nm}$ and $193 \mathrm{~nm}$ for the diamond, AlN and c-BN detectors, respectively.

For the diamond MSM24-r photodetector, the responsivity has a maximum of $48 \mathrm{~mA} / \mathrm{W}$ at $210 \mathrm{~nm}$ with corresponding EQE of $28 \%$. It displays a rejection ratio between $210 \mathrm{~nm}$ and $400 \mathrm{~nm}$ of more than four orders of magnitude $\left(1.610^{4}\right)$. The VUV measurement shows that MSM24-r photodetector sensitivity decreases rapidly towards $130 \mathrm{~nm}$ by a factor of 20 which is probably due to a combination of surface recombination, photoemission losses $[6,33]$ and the abrupt drop of the diamond penetration depth (cf. Fig. 1). The responsivity starts to increase slowly from $130 \mathrm{~nm}$ to $75 \mathrm{~nm}$ by a factor of 3 and becomes almost constant at higher photon energies which is related to an increase of the number of electron-hole $(\mathrm{e}-\mathrm{h})$ pairs created per absorbed photon.

The AlN MSM photodetector shows a rejection ratio between $200 \mathrm{~nm}$ and $360 \mathrm{~nm}$ of more than four orders of magnitude $\left(1.1510^{4}\right)$. Over the wavelength range of $170 \mathrm{~nm}$ to $200 \mathrm{~nm}$, the photoresponse is constant, in contrast to the diamond MSM24-r photodetector, but with a lower responsivity of approximately $4.5 \mathrm{~mA}$ /W at a bias voltage of $+30 \mathrm{~V}$. Then the photoresponse increases (depending on the polarity of the applied voltage) due mainly to the photoemission current contribution [18]. The photo-emitted electrons are pulled back to the device due to the high positive voltage of $+30 \mathrm{~V}$ applied. Thus, it is recommended biasing the electrical circuit to energies higher than the maximum kinetic energy that the electron can acquire after leaving the surface i.e. the photon energy minus the material's work function. At around $115 \mathrm{~nm}$, the photoresponse reaches a maximum responsivity of $28 \mathrm{~mA} / \mathrm{W}$ (with a corresponding EQE of 30\%). Then, it decreases until $80 \mathrm{~nm}$. This value is still close to the ionization threshold of almost any material meaning that the absorption is high with the generation of a high number of electrons close to the surface. Between $60 \mathrm{~nm}$ and $80 \mathrm{~nm}$, the responsivity is nearly constant with a photoresponse of $8.4 \mathrm{~mA} / \mathrm{W}$. For wavelengths shorter than $60 \mathrm{~nm}$, the responsivity starts to increase, related again to secondary ionisations. For AlN, the energy to create an electron-hole pair is predicted to be at $\lambda_{\mathrm{C}}=66 \mathrm{~nm}$, corresponding to $18.8 \mathrm{eV}$ [34] (13 eV for diamond [35]).

The maximum photoresponse of the c-BN MSM photodiode was observed at $180 \mathrm{~nm}$ with $21 \mathrm{~mA} / \mathrm{W}$ at $+30 \mathrm{~V}$ bias. The cut-off wavelength of the device is around $193 \mathrm{~nm}(6.42 \mathrm{eV})$ corresponding to

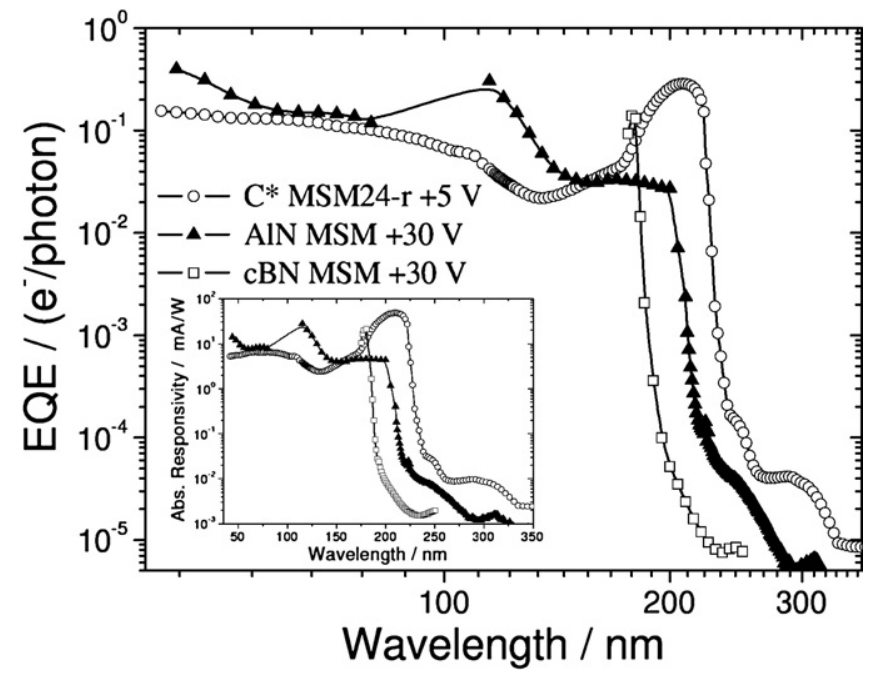

Fig. 6. External quantum efficiencies (EQE) of the diamond MSM24-r photodetector at $5 \mathrm{~V}$ bias, of the c-BN MSM photodiode at $+30 \mathrm{~V}$ bias (in the range between $175 \mathrm{~nm}$ and $250 \mathrm{~nm}$ ), and of the AlN MSM photodiode at $+30 \mathrm{~V}$ bias, in the wavelength range between $40 \mathrm{~nm}$ and $360 \mathrm{~nm}$. The inset shows the corresponding absolute spectral responsivity in the same wavelength range. 
Table 1

Derived results for the WBGM MSM photodetectors.

\begin{tabular}{|c|c|c|c|c|}
\hline Bold label & Parameters/applied bias & Max. response $(\mathrm{mA} / \mathrm{W})$ & Cut-off (nm) & Rejection ratio max response $/ 280 \mathrm{~nm}$ \\
\hline Diamond MSM24-r & s5w2/ + 5 V & $48(210 \mathrm{~nm})$ & $225(5.5 \mathrm{eV})$ & $510^{3}$ \\
\hline AlN MSM & $s 4 w 2 /+30 V$ & $4.5(190 \mathrm{~nm})$ & $203(6.1 \mathrm{eV})$ & $2.510^{3}$ \\
\hline cBN MSM & $s 5 w 2 /+30 v$ & $21.2(180 \mathrm{~nm})$ & $193(6.42 \mathrm{eV})$ & $1.0510^{4}$ \\
\hline
\end{tabular}

s, w: space and width of the interdigitated fingers.

the shortest value achieved for semiconductor detectors so far and agreeing well with the bandgap of the bulk c-BN $[36,37]$. The rejection ratio between $180 \mathrm{~nm}$ and $250 \mathrm{~nm}$ (noise limited) is around four orders of magnitude $\left(1.0510^{4}\right)$. The responsivity of the c-BN MSM photodiode was not yet measured in the VUV range but a synchrotron calibration campaign is planned in the future.

Table 1 reports the maximum responsivity of the MSM devices, as well as their threshold energies and their UV (280 nm) rejection ratio. Note that the UV rejection is measured as the ratio of the photoresponse and not of the EQE. Since EQE is wavelength dependent, it leads to an increased rejection value.

Further tests on the MSM photodetectors were performed (but not reported here), the signal stability, the linearity with photon flux, and the homogeneity of the response over the whole surface (raster scan) were measured. In the wavelength range of interest, the MSM photodetectors based on diamond, AIN and c-BN have proven to be stable on a wide temporal range, linear (at least over three orders of magnitude), and homogenous.

\section{Conclusions}

By their nature, diamond, AlN and c-BN semiconductors are the primary choice of photosensitive materials for VUV photon detection. They present figure of merit that is several orders of magnitude higher compared to silicon semiconductors, since they provide high radiation hardness, low dark signal at room temperature, solar-blindness, and chemical as well as thermal stability. The purpose of this work was to demonstrate the suitability of the new WBGM MSM photodetectors for solar observations on space missions, in order to have a solid detector baseline for VUV radiometers and imaging telescopes.

The MSM photodetectors based on diamond, AlN and c-BN were characterized in the NUV to the VUV range when possible. A sharp cutoff wavelength was observed at around 225, 203 and $193 \mathrm{~nm}$ corresponding to the bandgap energies of the diamond, AlN and c-BN bulk materials, respectively. In the wavelength range of interest, the detectors show extremely low dark current, high stability and good sensitivity. The rejection ratios between the maximum and the $280 \mathrm{~nm}$ response were measured to be better than 3 to 4 orders of magnitude.

Although these MSM devices proved their general suitability for space applications, diamond, BN and AlN must still be acknowledged as relying on new/emerging technology. Further improvements of their performance characteristics are still needed and can be anticipated. Other different design approaches to improve the responsivity of MSM photodetectors are investigated such as size reduction towards submicron contact fingers, semi-transparent electrodes, and/or asymmetric electrodes. All these solutions can provide better detector characteristics in terms of dark current, UV/ visible contrast ratio, linearity, and VUV responsivity. We expect advantages by operating the MSM photodetectors at high positive bias voltages maximizing the output signal but also reducing the electron escape probability in the VUV range.

\section{Acknowledgement}

The authors would like to thank the Belgian Federal Science Policy Office supporting this programme through the ESA-PRODEX. The research at KSU is supported by a grant from DOE. The work in COSDAF was funded by the Research Grants Council of the Hong Kong Special Administrative Region, China (Project No. City U 123607 and City U 123806).

\section{References}

[1] http://bold.oma.be

[2] M. Liao, Y. Koide, J. Alvarez, Appl. Phys. Lett. 90 (2007) 123507.

[3] A. De Sio, J. Achard, A. Tallaire, R.S. Sussmann, A.T. Collins, F. Silva, E. Pace, Appl. Phys. Lett. 86 (2005) 213504.

[4] A. Balducci, M. Marinelli, E. Milani, M.E. Morgada, A. Tucciarone, G. Verona-Rinati, M. Angelone, M. Pillon, Appl. Phys. Lett. 86 (2005) 193509.

[5] M. Nesladek, Semicond. Sci. Technol. 20 (2005) R19.

[6] T. Saito, K. Hayashi, H. Ishihara, I. Saito, Metrologia 43 (2006) S51.

[7] W. Adam, E. Berdermann, P. Bergonzo, W. de Boer, F. Bogani, E. Borchi, et al., Eur. Phys. J. C33 (2004) S1014

[8] M. Razeghi, A. Rogalski, J. Appl. Phys. 79 (1996) 7433.

[9] U. Schühle, J.-F. Hochedez, J.L. Pau, Proc. SPIE 5171 (2004) 231

[10] E. Monroy, F. Omnès, F. Calle, Semicond. Sci. Technol. 18 (2003) R33.

[11] D.R. Kania, M.I. Landstrass, M.A. Plano, Diamond Relat. Mater. 2 (1993) 1012.

[12] R.A. Roberts, W.C. Walker, Phys. Rev. 161 (1967) 730.

[13] D.L. Windt, W.C. Cash Jr., M. Scott, P. Arendt, B. Newman, R.F. Fisher, A.B. Swatzlander, P.Z. Takacs, J.M. Pinneo, Appl. Opt. 27-2 (1988) 279.

[14] D.F. Edwards, E. Ochoa, J. Opt. Soc. Am. 71 (1981) 607.

[15] W.C. Walker, J. Osantowski, Phys. Rev. 134-1A (1964) 153.

[16] http://henke.lbl.gov/optical_constants/.

[17] D.J. Jones, R.H. French, H. Mullejans, S. Loughin, A.D. Dorneich, P.F. Carcia, J. Mater. Res. 14-11 (1999) 4337.

[18] A. BenMoussa, J.F. Hochedez, R. Dahal, J. Li, J.Y. Lin, H.X. Jiang, A. Soltani, J.-C. De Jaeger, U. Kroth, M. Richter, Appl. Phys. Lett. 92 (2008) 022108.

[19] J.-F. Hochedez, W.K. Schmutz, M. Nesladek, Y. Stockman, U. Schühle, A. BenMoussa, S Koller, K. Haenen, D. Berghmans, J.-M. Defise, J.-P. Halain, A. Theissen, V. Delouille, V. Slemzin, D. Gillotay, D. Fussen, M. Dominique, F. Vanhellemont, D. McMullin, M. Kretzschmar, A. Mitrofanov, B. Nicula, L. Wauters, H. Roth, E. Rozanov, I. Rüedi, C. Wehrli, H. Amano, R. Van der Linden, A. Zhukov, F. Clette, S. Koizumi, V. Mortet, Z. Remes, R. Petersen, M. D'Olieslaeger, J. Roggen, P. Rochus, Adv. Space Res. 37-2 (2006) 303.

[20] Z. Remes, R. Petersen, K. Haenen, M. Nesladek, M. D'Olieslaeger, Diamond Relat. Mater. 14 (3-7) (2005) 556.

[21] A. BenMoussa, A. Soltani, K. Haenen, U. Kroth, V. Mortet, H.A. Barkad, D. Bolsee, C. Hermans, M. Richter, J.C. De Jaeger, J.F. Hochedez, Semicond. Sci. Technol. 23 (2008) 035026.

[22] W.J. Zhang, I. Bello, Y. Lifshitz, K.M. Chan, X.M. Meng, Y. Wu, C.Y. Chan, S.T. Lee, Adv. Mater. 16 (2004) 1405.

[23] W.J. Zhang, I. Bello, Y. Lifshitz, S.T. Lee, MRS Bull. 28 (2003) 184.

[24] W.J. Zhang, Y.M. Chong, I. Bello, S.T. Lee, J. Phys. D: Appl. Phys. 40 (2007) 6159.

[25] A. Soltani, H.A. Barkad, M. Mattalah, B. Benbakhti, J.-C. De Jaeger, W.J. Zhang, Y.M Chong, Y.S. Zou, S.T. Lee, A. BenMoussa, B. Giordanengo, J.-F. Hochedez, Appl. Phys. Lett. 92 (2008) 053501.

[26] J. Li, Z.Y. Fan, R. Dahal, M.L. Nakarmi, J.Y. Lin, H.X. Jiang, Appl. Phys. Lett. 89 (2006) 213510.

[27] J. Li, B. Nam, M.L. Nakarmi, J.Y. Lin, H.X. Jiang, Appl. Phys. Lett. 81 (2002) 3365.

[28] J. Li, B. Nam, M.L. Nakarmi, J.Y. Lin, H.X. Jiang, P. Carrier, S.H. Wei, Appl. Phys. Lett. 83 (2003) 5163.

[29] R. Dahal, T.M. Al Tahtamouni, Z.Y. Fan, J.Y. Lin, H.X. Jiang, Appl. Phys. Lett. 90 (2007) 263505.

[30] R. Dahal, T.M. Al Tahtamouni, J.Y. Lin, H.X. Jiang, Appl. Phys. Lett. 91 (2007) 243503.

[31] M. Richter, J. Hollandt, U. Kroth, W. Paustian, H. Rabus, R. Thornagel, G. Ulm, Nucl. Instr. Methods 467-468 (2001) 605.

[32] M. Richter, U. Kroth, A. Gottwald, C. Gerth, K. Tiedtke, T. Saito, I. Tassy, K. Vogler, Metrologia 40 (2003) S107.

[33] T. Saito, K. Hayashi, Appl. Phys. Lett. 86 (2005) 122113.

[34] C.A. Klein, J. Appl. Phys. 39 (1968) 2029.

[35] A. BenMoussa, A. Theissen, F. Scholze, J.-F. Hochedez, U. Schühle, W. Schmutz, K. Haenen, Y. Stockman, A. Soltani, D. McMullin, R.E. Vest, U. Kroth, C. Laubis, M. Richter, V. Mortet, S. Gissot, V. Delouille, M. Dominique, S. Koller, J.-P. Halain, Z. Remes, R. Petersen, M. D'Olieslaeger, J.-M. Defise, Nucl. Instrum. Methods A 568 (2006) 398.

[36] S.N. Mohammad, Solid State Electron. 46 (2002) 203

[37] R.M. Chrenko, Solid State Commun. 14 (1974) 511. 